\title{
La complejidad y opacidad de las tareas auxiliares en los procesos de trabajo: un análisis etnográfico comparado en dos servicios hospitalarios
}

\author{
Carlos LOZARES \\ Universidad Autónoma de Barcelona \\ Departamento de Sociología \\ carlos.lozares@uab.cat \\ Joan Miquel VERD \\ Universidad Autónoma de Barcelona \\ Departamento de Sociología \\ joanmiquel.verd@uab.cat \\ Oriol BARRANCO \\ Universidad Autónoma de Barcelona \\ Departamento de Sociología \\ oriol.barranco@uab.cat \\ Matilde MASSÓ \\ Universidad de la Coruña \\ Departamento de Sociología y CC. Política y de la Administración \\ m.masso@udc.es \\ Sara MORENO \\ Universidad Autónoma de Barcelona \\ Departamento de Sociología \\ sara.moreno@uab.cat
}

Recibido: 08-06-2011

Aceptado: 27-03-2012

\begin{abstract}
RESUMEN
En el artículo se analizan dos procesos de trabajo auxiliares de un hospital, como son el trabajo administrativo de las Consultas Externas y el trabajo del servicio de Esterilización. En concreto, el análisis se concentra en dos aspectos. El primero se refiere a la complejidad producida por el grado de trabajo interactivo de los procesos de trabajo. El segundo aspecto se refiere a la opacidad o invisibilidad del trabajo y de las competencias para realizarlo. La obtención de datos se ha realizado utilizando una orientación etnográfica, combinando el uso de observaciones y de entrevistas semidirigidas. El análisis se desarrolla repasando descriptiva e interpretativamente cada uno de los dos procesos y desplegando reflexiones comparativas entre ellos. El artículo se finaliza con conclusiones respecto a las características particulares que representan para los trabajadores y la propia organización las tareas de carácter auxiliar.
\end{abstract}


Palabras clave: etnografía en el trabajo, complejidad, trabajo auxiliar, trabajo invisible, competencias

Complexity and opacity of auxiliary tasks in work processes:
a comparative ethnographic approach in two hospital services

\begin{abstract}
In the article, two auxiliary work processes in a hospital are analysed; namely, the administrative work in the out-patients department and the work in the sterilisation service. The analysis focuses on two aspects. The first one refers to complexity produced by the degree of interactive work in work processes. The second one refers to opacity or invisibility of work and competencies necessary to accomplish it. Data has been obtained using an ethnographic approach, combining the use of observations and semi-directed interviews. The analysis develops reviewing descriptively and interpretatively each work process and deploying comparative reflections among them. The article finishes with conclusions related to the particular characteristics that auxiliary tasks have for workers and the organisation itself.
\end{abstract}

Keywords: work ethnography, complexity, auxiliary work, invisible work, competencies

\title{
REFERENCIA NORMALIZADA.
}

Lozares, C., Verd, J. M., Barranco, O., Massó, M. y Moreno S. (2013). La complejidad y opacidad de las tareas auxiliares en los procesos de trabajo: un análisis etnográfico comparado en dos servicios hospitalarios. Cuadernos de Relaciones Laborales. Vol. 31, núm. 1, p. 221-242.

Sumario: Introducción. 1. Referencias teóricas y conceptuales. 2. La metodología y los métodos de recogida de información. 3. Las características del hospital y de los servicios estudiados. 4. El análisis y la interpretación. 5. Reflexiones y conclusiones. 6. Bibliografía.

\section{Introducción}

Presentamos en este artículo un análisis de dos procesos de trabajo que se enmarcan en dos servicios diferentes de un hospital público generalista. Concretamente, se estudian el trabajo administrativo de Consultas Externas y el trabajo de Esterilización. Tanto el trabajo administrativo como el de esterilización tienen un carácter auxiliar con respecto a otras tareas médicas y quirúrgicas hospitalarias. El hecho de situarse ambos procesos de trabajo en el mismo hospital ha facilitado su comparación dentro del mismo contexto institucional, médico y social.

Los objetivos de este artículo son parte de los abordados en una investigación más amplia sobre los procesos de trabajo ${ }^{1}$, concretamente sobre su naturaleza y función, las interacciones entre agentes e instrumentos, los componentes cognitivos (representación, memoria, automatización, información, decisión...) que se generan e incorporan al trabajo y sobre la presencia e influencia de los diferentes contextos. Se partía del supuesto de que tales fenómenos son componentes y factores decisivos

${ }^{1}$ Contrato SEC2001-2543 del Grupo de Estudios Sociológicos sobre Vida Cotidiana y Trabajo (QUIT) de la UAB. 
de la evolución y modificación de los procesos de trabajo. En este artículo nos centramos, concretamente, en el análisis de dos aspectos. El primero se refiere a la complejidad, que es fruto tanto del número de agentes, interacciones e instancias que intervienen en el desarrollo de tareas y funciones, como de la simultaneidad y fraccionamiento de los procesos de trabajo. El segundo aspecto tiene que ver con la opacidad o invisibilidad del trabajo y con las competencias aplicadas en su ejercicio. Ambos aspectos, complejidad y opacidad están vinculados entre sí y son reveladores de los momentos de transición y cambio en los procesos de trabajo, concretamente, en el caso en los servicios hospitalarios.

Estas temáticas y objetivos se desarrollan en cuatro apartados. En el primero, se señalan las referencias teóricas y conceptuales en que se fundamenta el artículo. En el segundo, se describe la metodología de recogida de datos. En el tercero, se presentan brevemente los dos servicios estudiados. En el cuarto, se analizan e interpretan los procesos de trabajo en ambos servicios a partir de los objetivos y temáticas planteados, tanto individual como comparativamente. Por fin, en el quinto apartado, se sintetizan las reflexiones y conclusiones más relevantes.

\section{Referencias teóricas y conceptuales}

Es lugar común y una evidencia constatada afirmar que los procesos de trabajo han sufrido en las últimas décadas cambios notables y rápidos que provienen de exigencias vinculadas a factores globales financieros y de mercado, laborales, políticos, etc. Tales cambios han repercutido y siguen repercutiendo de manera muy directa en la organización productiva de las empresas, de modo que la adaptación a dichas exigencias es ineluctable como una cuestión de supervivencia.

Pero estas transformaciones no son sólo de tipo global sino también de orden más elemental y cercano a los procesos directos de trabajo, que afectan a la vida cotidiana del/a trabajador/a en la empresa y fuera de ella, como por ejemplo, la eventualidad, temporalidad e inseguridad en el empleo, la disponibilidad y dependencia temporal, espacial y vital del trabajo productivo, el reciclaje profesional o la ausencia de conciliación temporal familiar. Otros cambios pueden ser vistos como benéficos para los/as trabajadores/as como la presencia, densidad y gestión del capital cognitivo; la información, automatización y computación; el trabajo en equipos cooperativos, comunidades de prácticas; el funcionamiento participativo y distribuido y de capital relacional; la autonomía, responsabilidad, autoaprendizaje, reciclaje y autoevaluación; el trabajo virtual y a distancia, movilidad temporal y espacial; las competencias (conocimientos, actitudes y pericias) flexibles y adaptativas... Los cambios pueden tener también efectos ambivalentes o perversos para los/as trabajadores/as, tanto por la manera de implementarlos al imponerse de manera imperativa y normativa desde la dirección sin conocimiento, consenso o adaptación por parte de las personas implicadas, como por no tener en cuenta en su evaluación y aplicación el carácter situacional, interactivo y contextual de los procesos de trabajo sobre todo si afectan a la inserción, promoción o salario. 
El sector público, ya se trate de empresas o de administraciones, no escapa a las tendencias que acabamos de señalar. En los últimos veinte años, a través de los programas de la denominada Nueva Gestión Pública, se vienen aplicando diversos modos de reestructuración con el objetivo explícito de hacer más flexible la organización y el sistema productivo y hacer más eficiente el sector público (Dunleavy, 1994; Hood, 1994; Barzelay, 2001; Gallego, 2002). Consisten básicamente en trasladar al sector público modelos y método de gestión que se vienen aplicando en la empresa privada (Olías de Lima, 1999) ${ }^{2}$. Por ello, y en lo que se refiere a su gestión y organización, la empresa pública y privada tienden a equipararse.

Del conjunto de toda la amplia problemática expuesta, este artículo se refiere exclusivamente a dos aspectos específicos que tienen que ver con los cambios señalados. En primer lugar, se trata de identificar y comprender la naturaleza compleja de los procesos de trabajo, particularmente en lo que se refiere a las interacciones entre agentes y de ellos con los instrumentos así como la implicación de la instancia cognitiva que ello supone. En segundo lugar, el artículo se refiere a otros dos aspectos vinculados entre sí: a las competencias o capacidades correspondientes que suponen dichos cambios en los/as trabajadores/as y que frecuentemente quedan ocultos tanto para los propios trabajadores como para la organización. Se entiende que la invisibilidad no se puede desvincular de la influencia que ejerce la división sexual del trabajo, si bien el artículo fija su interés en los factores procesuales indicados.

El marco teórico que ha orientado la investigación general en que se inscribe este artículo se encuentra más ampliamente desarrollado en Lozares et al. (2004). Con todo, se señalan a continuación las orientaciones teóricas más directamente vinculadas a los objetivos y temáticas concretos indicados anteriormente.

El primer referente tiene que ver con la intensificación de los procesos de trabajo, y más concretamente en lo que se entiende como flujo tenso (Castillo et al., 2000; Durand, 2001, 2004; Pinilla, 2004). Una organización productiva basada en la idea de flujo tenso supone la máxima reducción de los tiempos de recuperación o de transición entre tareas, es decir, la supresión de la porosidad del trabajo. En estos casos se incrementa la intensidad del trabajo no exclusivamente por medio de la aceleración de los ritmos, sino mediante una reorganización del trabajo que básicamente trata de eliminar los tiempos vacíos. Tal como señala Durand (2001), el principio básico es el de construir un flujo continuo de materia (o de informaciones, o de consumidores, o de usuarios) del cual los trabajadores sean los responsables más directos. Frente al modelo taylorista, en que los ritmos de trabajo son externamente controlados, en el modelo de flujo tenso es el/la propio/a trabajador/a quien establece su propio ritmo, pero siempre en un contexto de urgencia; cada uno/a es responsable de organizar lo más eficientemente su propio tiempo, siempre teniendo en cuenta que los tiempos de espera deben ser los mínimos.

\footnotetext{
${ }^{2}$ Véase Pastor $(2004 ; 2008)$ para el caso de los servicios sanitarios en Catalunya.
} 
El segundo referente teórico se centra en la gestión de los proceso de trabajo, particularmente en lo que se denomina gestión por competencias. Autores como Lichtenberger (2003), Sala y Planas (2009) o Massó (2011) señalan que, en una organización productiva basada en la gestión por competencias la valoración de la eficiencia del trabajador/a, no sólo se calibra por sus cualificaciones o credenciales sino por las habilidades, actitudes, aptitudes profesionales, sociales y humanas puestas en juego en la situación de la práctica del trabajo, tarea o actividad. Este modelo de gestión suele conllevar la ausencia de negociación colectiva o global de la empresa con los agentes sociales ya que se prioriza la negociación individual con el trabajador, al menos para una parte de sus beneficios o remuneraciones. En este contexto, se prima también la implicación en la empresa de los/as trabajadores/as en todos los niveles; es decir, se trata de poner al servicio de la organización productiva (Paradeise y Lichtenberger, 2001), lo que podría llamarse una "inteligencia global" pero negociada individualmente.

El tercer referente consiste en la invisibilidad que se da en los procesos de trabajo de determinadas tareas, y que las transformaciones actuales de la organización del trabajo tienden a incrementar. Tres aportaciones destacan al respecto. Una primera proviene de la perspectiva de género. Desde ésta se subraya, por un lado, que las mujeres suelen ocupar lugares de trabajo menos visibles que los hombres dada la división sexual del mercado laboral y de las tareas dentro de las organizaciones (Kergoat, 1994; Crompton, 1997; Messing, 1998); y, por otro, se destaca que muchas de las cualificaciones o competencias de las mujeres se mantienen como invisibles, sea porque son adquiridas por canales institucionales no reconocidos (Kergoat, 1982) o porque se consideran innatas, propias de la naturaleza femenina (Kergoat, 1982; Elson y Pearson, 1981). Se trata de cualificaciones o competencias que, con frecuencia, se adquieren en el ámbito doméstico a través de la socialización diferencial de género $\mathrm{y}$, por ello, vistas como consustanciales al género femenino. De este modo, los empleos que demandan estas cualificaciones o competencias consideradas naturales en las mujeres suelen entenderse socialmente como no cualificados o poco cualificados precisamente a causa de su invisibilidad (Elson y Pearson, 1981; Jenson, 1989). Una segunda aportación es la de De Terssac (1995), que señala que en la medida en que en la organización del trabajo se han ido relajando las exigencias fijadas por las reglas formales, aparece un margen de autonomía en que se realizan tareas que para la empresa pasan desapercibidas. Este trabajo invisible consiste en el conjunto de obligaciones y actividades implícitas o no visibles pero efectivas que se realizan en el ejercicio de las tareas y por tanto no explicitadas. Se trata, pues, de prácticas no prescritas, pero de alguna manera asumidas como incorporadas al proceso de trabajo y que procuran su mejor desarrollo en las condiciones reales de la producción. Por último, una tercera aportación es la de Arborio (2001) al señalar que el carácter invisible del trabajo auxiliar en los hospitales está relacionado con el hecho de que lo realizan mujeres, que no constituyen un grupo unitario dada la variedad de prácticas y procesos que se engloban bajo una misma categoría administrativa. 
El cuarto referente teórico tiene que ver con las perspectivas de la actividad situada y del conocimiento socialmente distribuido (Conein, 1994; Suchman, 1993). Desde los presupuestos de dichas concepciones, para identificar y analizar los procesos de trabajo y sus cambios se ha de considerar como unidad más elemental de análisis y de significado las interacciones entre los trabajadores, entre sí y con los artefactos, y no sólo ni prioritariamente la acción exclusiva y aislada de cada trabajador fuera de su ejercicio en situación y contexto. Por otro lado, se ha de considerar el peso decisivo de la incidencia de la instancia cognitiva en los procesos de trabajo en la que se incluye su externalización en las pantallas, protocolos, conversación, resultados, etc. De esta manera, y dada la unidad de referencia tomada, la cognición aparece además distribuida y puede así ser compartida y objeto de acuerdo entre los agentes intervinientes. Por fin, se ha de tener también en consideración la importancia de la intervención de los artefactos en los procesos de trabajo. En este sentido, no se puede establecer una dicotomía entre acciones comunicativas, cognitivas y acciones instrumentales a partir de la distinción entre agentes humanos y los instrumentos de trabajo. Toda práctica interactiva proviene también y está mediatizada por los artefactos, máxime en los procesos de trabajo actuales: sistemas notacionales, automatización, mecanismos de información y cálculo, robotización, tecnologías cada vez más "inteligentes" (Hutchins, 1995; Hutchins y Klausen, 1996; Lozares, 2000, 2001, 2007a, 2007b).

\section{La metodología y los métodos de recogida de información}

Como se ha señalado, los dos procesos de trabajo estudiados, aunque muy diferentes entre sí, tienen un carácter auxiliar respecto a otros procesos más generales como los de atención médica o intervención hospitalaria. El hecho de que pertenezcan al mismo hospital favorece su comparabilidad, puesto que los contextos estructurales en que se desarrollan son idénticos. El objetivo consiste precisamente en verificar las consecuencias que, en procesos de trabajo no centrales, tiene el ejercicio de una mayor responsabilidad individual y de una menor prescripción detallada inspirada por la lógica de la competencia. Al mismo tiempo, se pretende comprobar si la desaparición parcial de protocolos minuciosos e imperativos incrementa o no el grado de complejidad y el nivel de invisibilidad en el desempeño de las tareas.

El tipo de aproximación metodológica realizada en la investigación se inspira genéricamente en la etnografía de los procesos de trabajo, y más concretamente en las investigaciones realizadas por Cicourel en entornos médicos $(1999,2007)$. Ello implicaba situar en un lugar central la técnica de la observación, utilizando tanto notas de campo como la grabación con videocámara. Con todo, la obtención de datos mediante observación se articuló con el uso de entrevistas semidirigidas de contenido narrativo-profesional aplicadas a los/as trabajadores/as y otras de tipo informativo realizadas a personas con grados diferentes de responsabilidad en el hospital, junto al análisis documental para contextualizar y tener información de las áreas y los procesos de trabajo estudiados. Esta orientación metodológica es la más 
pertinente, por un lado, para la identificación, análisis e interpretación de nuestro objeto de estudio, como es la complejidad e invisibilidad de los procesos de trabajo $\mathrm{y}$, por otro, para el carácter microprocesual, situacional y contextual de dichos procesos. ${ }^{3}$ Por otro lado, y dado el carácter comparativo de nuestro estudio entre dos servicios, la elección del diseño metodológico y de los procedimientos de recogida de datos y de análisis debían ser semejantes pero con suficiente flexibilidad como para adaptase a las diferencias entre ambas situaciones y permitir su comparación. En este sentido, se han seguido los consejos de Peneff (1998) respecto al uso de la observación en contextos de trabajo. Este autor defiende una aproximación con un marcado carácter inductivo por lo que la teoría funciona más como generadora de preguntas de investigación y no tanto como "plantilla de observación" (Peneff, 1998: 12-17).

En el servicio de Consultas Externas la observación se desarrolló en dos salas de espera tomadas como representativas del conjunto de servicio (Verd, Barranco y Moreno, 2007). Además, se hicieron entrevistas semidirigidas a las administrativas de dichas salas. En Esterilización la observación se llevó a cabo en todos los procesos del servicio y las entrevistas a todas las personas involucradas.

La observación planteada fue de tipo abierto y no participante. En una primera fase, tuvo un carácter exploratorio, distante y general sobre todo el proceso de trabajo, tanto para que las personas observadas se habituasen a la presencia de observadores como para que éstos tomaran en cuenta la totalidad del proceso, eligieran los mejores espacios para las observaciones siguientes (sobre todo las realizadas con vídeo) y conocieran a las personas y sus funciones. En esta fase se tomaron notas de campo genéricas. En una segunda fase se llevó a cabo una observación también distante y con notas de campo minuciosas captando la totalidad de los procesos. En una tercera fase, con mayor confianza recíproca con los observados, la observación se realizó por videocámara acompañada por notas de campo. Las cámaras se mantuvieron en posición fija o móvil (manipuladas por los observadores) en función de las necesidades del proceso de observación y de las características particulares de cada uno de los servicios. En los tres tipos de observación, cada proceso y subproceso fueron observados por diferentes personas y en días diferentes, al menos dos veces.

\section{Las características del hospital y de los servicios estudiados}

\subsection{El hospital}

El hospital donde se desarrolló el estudio está ubicado en Catalunya. Forma parte y está gestionado por un organismo autónomo público, que hemos denominado

\footnotetext{
${ }^{3}$ Dichos conceptos objeto de estudio serán más detallada y explícitamente definidos en el aparado 4 dedicado al análisis e interpretación.
} 
para mantener su anonimato "Instituto Sanitario" y que integra también a otros centros sanitarios de distinta índole. Este Instituto Sanitario forma parte de la administración pública, pero sus órganos gestores disponen de una gran autonomía de gestión.

Los servicios de Consultas Externas y de Esterilización, donde se ha realizado la investigación, se ubican dentro del hospital. Dado el carácter generalista y público, el hospital trata la mayor parte de las patologías que puede padecer su población de referencia. Es un hospital de dimensión relativamente grande como lo muestra su plantilla de personal fijo (excluyendo el personal eventual y el de los servicios subcontratados) de más de un millar de personas, las 400 camas que dispone y el área de influencia con una población de referencia de alrededor de 500.000 habitantes.

\subsection{El servicio de Consultas Externas}

En el servicio de Consultas Externas se atiende a los pacientes no internados en el hospital. En el trabajo administrativo de este servicio se llevan a cabo tres procesos de trabajo diferenciados: la atención a los usuarios/as, la petición/repesca de historiales médicos y la facturación. Los tres procesos están estrechamente vinculados entre sí. El primero incluye tareas de atención al público y los otros dos suponen tareas administrativas. La atención al usuario/a consiste en recibir al paciente, informarse de su solicitud o petición correctamente, dar hora para las pruebas médicas y el día de visita con el médico/a. El proceso de historiales médicos consiste en proporcionar al médico, para el día de la visita, el historial del paciente y el resultado de las pruebas realizadas. Y, finalmente, la facturación supone la contabilidad y registro de la visita. Se deduce, pues, que el proceso de trabajo administrativo de Consultas Externas está vinculado a distintos servicios del hospital: atención médica, archivo general y diferentes servicios hospitalarios dónde se realizan pruebas médicas.

\subsection{EI servicio de Esterilización}

El servicio de Esterilización se sitúa y se gestiona también en y desde el Hospital. La esterilización se lleva a cabo sobre el instrumental quirúrgico por procedimientos físicos como la presión, temperatura y humedad y, en algunos casos, por procedimientos químicos. Se esterilizan instrumentos y materiales, reciclables (lienzos, gasas...), reutilizables y no desechables que provienen de quirófanos, maternidad, consultas, camas, etc. del hospital y se redistribuyen en los centros de origen. En este proceso general se pueden considerar diferentes procesos. Primero, el 'prelavado-lavado' que contiene a su vez diferentes fases: el remojo y prelavado del instrumental, la colocación e introducción del instrumental en las lavadoras, el funcionamiento y control de las mismas y la extracción del instrumental de las lavadoras. Segundo, el que se denomina 'hacer cajas' que consiste en la introducción del instrumental en cajas para su esterilización. Está compuesto de las fases siguientes: el despliegue de un lienzo sobre la mesa, colocación de instrumentos sobre el lienzo, introducción de los instrumentos en caja, su control, cierre, precinto, registro e identificación. Tercero, el de 'doblar lienzos', que consiste en el pliegue de tejidos 
(batas, lienzos, gasas) y la formación de paquetes para su esterilización. Está constituido por diferentes fases: el pliegue de lienzos, la preparación y disposición en hatos, la introducción en ellos de una cinta de control de esterilización, el empaquetado con papel esterilizado, y, por fin, el cierre, identificación y registro del paquete. Cuarto, en primer lugar, el de la esterilización del propio autoclave y, en segundo lugar, del instrumental en el autoclave. Esta fase consiste en la introducción de las cajas y paquetes en el autoclave, su puesta en marcha, el control del proceso y la extracción y distribución de cajas en los quirófanos y maternidad y de los paquetes en la sala esterilizada. Quinto, la distribución externa del material a otros servicios del hospital.

\section{El análisis y la interpretación}

A continuación se exponen los análisis e interpretaciones a partir de los conceptos básicos objeto de nuestro estudio en cada uno de los servicios. Al inicio de cada uno de los apartados 4.1. y 4.2. se da una definición descriptiva de tales conceptos y objeto de estudio que sirven como guía genérica en el análisis e interpretación siguiendo la línea inductiva adoptada.

\subsection{La complejidad de los procesos de trabajo}

La complejidad referida a un proceso de trabajo se identifica a partir de la presencia y de la manera en que se articulan entre sí un conjunto de elementos o componentes que intervienen en los procesos de trabajo. En la medida que tales elementos concurran con más extensión, intensidad, copresencia y dinámica, tales procesos son más complejos:

(i) número de agentes, artefactos y tareas;

(ii) interacciones entre agentes $\mathrm{y} / \mathrm{o}$ artefactos simultáneas, paralelas o retroactivas como opuesto a únicas y lineales;

(iii) conjunción de instancias de diferente naturaleza como son las funcionales, cognitivas y sociales así como presencia e intervención de contextos,

(iv) exigencia de polivalencia de competencias en los procesos

(v) interrupciones $\mathrm{y} / \mathrm{o}$ perturbaciones en el proceso en función de factores imprevisibles o no programados

(vi) resultados emergentes más allá de una simple adición lineal de componentes.

4.1.1. Los espacios, la organización, la cooperación, distribución de tareas y capacidad auto-organizativa en los procesos de trabajo contemplados como factores de complejidad

\section{En las Consultas Externas}

El equipo que realiza el trabajo administrativo por la mañana en las Consultas Externas lo forman dieciséis personas: una coordinadora y quince auxiliares admi- 
nistrativos/as. De las quince, catorce son mujeres y sólo uno es hombre; once trabajan en las salas de espera, cada uno/a en la sala que le ha sido asignada y los cuatro restantes lo hacen en la recepción. Nuestro análisis se ha centrado únicamente en el proceso de trabajo de las auxiliares administrativas de las salas de espera.

La ubicación de las auxiliares administrativas y el diseño de su trabajo están relacionados con la organización general de las Consultas Externas. Estas últimas se distribuyen en once salas de espera, cada una de las cuáles está compuesta por seis consultas o boxes de médicos especialistas. Para cada dos boxes suele haber una enfermera o una auxiliar de enfermería y para atender a los usuarios/as de los seis boxes que componen la sala de espera hay una auxiliar administrativa. Así, cada auxiliar administrativa de sala queda fijada a una sala y a los equipos médicos (médicos, enfermeras y secretarias médicas) que en ella trabajan.

El diseño del espacio de trabajo de las auxiliares administrativas sitúa a éstas, por un lado, frente a los usuarios/as -puesto que la atención a éstos es su principal tarea-, y por el otro, al servicio de las enfermeras y médicos, quedando patente que sus tareas son auxiliares del proceso de trabajo médico. Sería muy diferente un diseño en el cual las administrativas se situasen en la recepción de Consultas Externas. La "descentralización" y "distribución" de las administrativas hacia las diferentes salas de espera, una para cada sala, permite que las enfermeras puedan interrumpir el trabajo de la administrativa sin alejarse de sus tareas en los despachos de consultas. Este diseño responde claramente a las necesidades de los procesos de trabajo de las enfermeras y los médicos, puesto que no tienen que desplazarse lejos del despacho de consultas en busca de un determinado servicio o una determinada información. De hecho, un anterior diseño del espacio y de la organización del trabajo administrativo ubicaba a las administrativas de las diferentes especialidades juntas en un único mostrador y una al lado de la otra. Ello permitiría la relación entre ellas, la cooperación, la resolución de ciertas dudas y problemas como las sustituciones y el aprendizaje mutuo y evitar el aislamiento durante la jornada. En cambio, la actual organización del trabajo y el diseño del espacio implica que las administrativas realicen individualmente los tres procesos de trabajo en el mismo puesto de trabajo de la sala: cada administrativa trabaja continuamente de cara al público, sin tener un espacio reservado para los procesos de trabajo que no son de atención al usuario/a (los mencionados de repesca de historiales y la facturación) y, además, sin poder compartir las tareas con nadie más.

Cada administrativa tiene la responsabilidad de realizar los tres procesos de trabajo ya enunciados durante la jornada laboral autorganizándose del mejor modo que pueda. Prácticamente la única indicación que las administrativas tienen respecto a cómo deben organizarse en la realización de sus tareas es la prioridad del proceso de atención al usuario respecto a los otros dos procesos de trabajo de repesca de historiales y de facturación. Por ello, dado que no existe ni protocolo ni unas indicaciones sobre cuándo realizar las tareas de facturación o repesca de historiales, éstos se realizan en los espacios vacíos que deja la atención a los usuarios, a veces incluso superpuestos con otros. Así, a primera vista puede parecer que las auxiliares administrativas disponen de una gran autonomía en la organización de su trabajo, 
puesto que está poco protocolado. Sin embargo, se trata de una autonomía muy limitada: en realidad no hacen el trabajo cómo y cuando quieran hacerlo sino cómo y cuando pueden en función de los flujos de demandas médicas y de los usuarios. De ahí que pueda decirse que se trata de un diseño organizativo que descansa en la auto-organización impuesta. Se responsabiliza a las administrativas de la organización de su propio trabajo, dejándoles un espacio para la autonomía individual, pero siempre dentro de los parámetros de control y subordinación a los usuarios/as, a los médicos y enfermeras. El resultado es que sin aumentar su capacidad de decisión y gestión autónoma sí que aumenta su estrés o carga mental, su aislamiento y la presión y tensión que soportan.

En este tipo de organización de las tareas, los aspectos más relevantes de la complejidad desde el punto de vista de la organización general del trabajo se refieren a la variedad de tareas, a la tensa presión del flujo de trabajo, a las urgencias, interrupciones y simultaneidad de las tareas y a los consecuentes estreses físicos y cognitivos.

\section{El servicio de Esterilización}

El equipo de esterilización de mañana está constituido por siete personas, todas mujeres, de las que presencialmente trabajan habitualmente seis, además de la encargada o coordinadora del equipo. De ellas, dos son enfermeras pero con tareas que no son propias de su cualificación, tres ayudantes de enfermera, con atribuciones consideradas adecuadas a su cualificación, dos de ellas con un cierto reconocimiento con relación al resto y una aprendiz trabajadora en prácticas; la coordinadora es enfermera y tiene gran experiencia en otros servicios hospitalarios. Los equipos trabajan por parejas y cada semana cambian de personas en cada pareja y tareas o procesos ya descritos. La coordinadora no sólo controla y orienta sino que participa de diversas tareas según necesidades. Dicha redistribución y otros temas vinculados al trabajo según necesidades y situaciones diarias o semanales, se deciden en uno de los momentos clave de comunicación diaria como es el desayuno conjunto, aparte de hablar de otros temas diferentes a los del trabajo.

El servicio de Esterilización se acerca más a la idea de un sistema complejo por su naturaleza más interactiva, por la presencia y variación de diversos actores y tareas, por la existencia de artefactos de diversa capacidad autónoma, así como por la mayor incidencia de la componente cognitiva y un grado de distribución social de tareas. Con todo, su carácter de "cápsula felizmente autogestionada" se ve empañada cuando se contextualiza el servicio en un marco más amplio. Primero, dado su relativo aislamiento de otros servicios y del "cara a cara" hospitalario con pacientes, visitas u otro/as trabajadores/as, se convierte en alguna situación observada en refugio (o "exilio") para trabajadoras, en el caso, no adaptadas a otro tipo de tareas o puestos de trabajo en el hospital; segundo, no obstante el grado de trabajo cooperativo, las responsabilidades, con posibles consecuencias importantes dado el tipo de tareas, son registradas, precintadas y guardadas de manera individualizada; tercero, el sistema de evaluación por competencias que comienza a introducirse de 
manera imparable conlleva una valoración subjetiva y de promoción individualizada en reconocimiento, salario y promoción.

\section{Consecuencias comparativas}

Cada uno de los servicios, Consultas Externas y Esterilización, han experimentado cambios de tipo organizativo tanto de naturaleza técnica como social. Se supone que estos cambios van en la dirección de una mayor productividad y rentabilidad de las competencias individuales y colectivas sin menoscabo del bienestar, motivación y beneficios individuales. Por lo constatado, cada servicio tiene resultados contradictorios e incluso efectos perversos consecuentes. Veamos estos aspectos comparativamente.

La organización de los procesos en Esterilización se asemeja a los de una cadena productiva clásica; sin embargo, no conlleva, primero, que las trabajadoras estén fuertemente sometidas a la presión de flujo salvo en situaciones de urgencias ni, segundo, que el trabajo sea muy determinista o intervencionista de manera directa y constante por contextos externos no obstante estar muy protocolado. Por el contrario, los procesos de Consultas Externas, que no son en cadena sino individualizados, están más sometidos a la presión tensa del flujo de la cola constante de los pacientes y de las urgencias que imponen las tareas que han de hacerse en simultáneo.

En Esterilización, las interacciones y presencias mutuas entre trabajadoras y la naturaleza cooperativa del trabajo son imprescindibles y promovidas, aunque más en unos procesos que en otros. Sin embargo, se presenta la contradicción de que a pesar de ello las responsabilidades de los procesos son atribuidos a la(s) persona(s) que cierran los procesos y los registros. Por el contrario, los procesos de Consultas Externas son de naturaleza individual y autónoma en su gestión, sin posibilidad de consulta gestual, visual u oral y sin el mínimo relajamiento comunicativo, además de consistir de tareas superpuestas con resolución inmediata y, evidentemente, con una responsabilidad individual directa.

El estrés físico, síquico y cognitivo y el absentismo se dan en los dos servicios, pero, sin duda, más en Consultas Externas, teniendo sólo en cuenta la naturaleza de las tareas, su ritmo y superposición. Las consecuencias diferentes en cuanto al aprendizaje, sustituciones, planificadas o imprevistas, son entonces evidentes y diferentes en uno u otro servicio.

\subsubsection{El grado y el tipo de simultaneidad de tareas y las interrupciones en el trabajo}

\section{Las Consultas Externas}

En las Consultas Externas la fuerte interrelación de los tres procesos de trabajo descritos (atención, historiales y facturación) se pone de manifiesto con el desarrollo de algunas tareas, parcial o completamente superpuestas temporalmente, lo que también supone la incidencia de diversas funciones e instancias cognitivas. Esta simultaneidad no quiere decir que sea total, se pueden dar en cortas dicotomías de secuenciación y simultaneidad, lo que no quita según el ritmo en que se produzca que sigan siendo procesos complejos. La dinámica del trabajo administrativo con- 
lleva continuas interrupciones en las tareas, a veces forzadas, por su complejidad, a la repetición de las mismas. La falta de protocolos explícitos para la regulación de los procesos junto a la dinámica implícita en las responsabilidades de las administrativas, a veces más allá de las estrictas a la administración (como por ejemplo las vinculadas a la gestión de las emociones de los pacientes), puede dar cuenta del grado y tipo de complejidad que conllevan. Con todo, la tarea centrada en el usuario/paciente es lo que marca el proceso principal que da linealidad a todo el conjunto asegurado además por el hecho de que las observaciones se realizaron durante el horario de atención al público.

\section{El servicio de Esterilización}

Los procesos de esterilización se caracterizan por una programación relativamente predeterminada, pues han de estar programados, protocolados y controlados dadas las exigencias del tipo de trabajo y su finalidad. Su desarrollo es lineal y serial aunque más autónomos y personalizados que las cadenas clásicas de producción. Con todo, se dan interrupciones y cambios de programación que provienen de otros servicios, en particular de quirófanos y, casi siempre, por la vía de urgencias.

Todo lo que es prelavado y lavado es serial, muy manual, de una realización relativamente simple y no necesariamente comunicativa e interactiva. Una ligera complejidad se introduce por el hecho de manejar a veces aparatos delicados o sensibles que requieren tipos específicos de manipulación.

La complejidad se hace más visible en el trabajo de control e identificación de los instrumentos y en el cierre y precinto de cajas y en la misma esterilización en sí. En este proceso, las trabajadoras operan en simultáneo con diferentes tipos de tareas e interacciones, como por ejemplo la comunicación con la persona que trabaja en otra caja, enfrente y en paralelo, al preguntarle por algún instrumento descolocado; la consulta de la trabajadora a unas guías-memos ad hoc donde se indican los instrumentos que han de ir en cada caja; la distribución sobre la mesa y la introducción en la caja de esterilización de dichos instrumentos en el orden que dicta el quirófano; los diferentes tipos de representaciones simultáneas que intervienen en la acción, etc. Añádase el grado de responsabilidad e implicación cognitiva que supone la identificación de cada caja, con el nombre propio de la trabajadora que lo ha controlado y cerrado con diversos tipos de registros y etiquetas, además de introducir otros controles de esterilización.

De una menor complejidad es el proceso de preparación de lienzos para su esterilización que se lleva a cabo en pareja. Pero puede darse alguna interrupción puesto que se compagina con la tarea de atender- inesperadamente por su momento aunque previsible en un lapso amplio del tiempo de la mañana- el trabajo de distribución del material esterilizado a las personas de otros servicios que vienen a solicitarlo. En esta situación una de las trabajadoras de la pareja interrumpe el trabajo en lienzos y acude a distribuir el material.

\section{Consecuencias comparativas}


Se dan diferencias sobre el carácter simple o complejo de ambos procesos de trabajo. Por un lado, en el caso de Consultas Externas se observa que las tareas dependen del flujo del conjunto del proceso, es decir, vienen marcadas por los usuarios/as o las demandas médicas, lo cual coincide con la definición de flujo tenso (Durand, 2001, 2004). En cambio, en el caso de Esterilización se observa un proceso de trabajo más bien continuo, ya que las tareas se vinculan unas a las otras y dependen de la organización técnico-productiva. Con todo, en algunas circunstancias pueden darse interrupciones o presiones fuertes por las demandas de esterilización de materiales y útiles. Por lo tanto, y en contraste con Consultas Externas, el ritmo de trabajo de Esterilización está más condicionado por el tiempo propio de cada máquina y por la cadencia de los "insumos" que llegan del quirófano y maternidad. En las Consultas Externas, el flujo tenso está introducido por las exigencias sincrónicas del ritmo de su trabajo propio.

En Esterilización el ritmo de trabajo es más regular en el desarrollo de las tareas, aunque, como se ha señalado, puede haber excepciones como es el caso de doblar la ropa y en los procesos que tengan exigencias contextuales intempestivas como las que pueden provenir de quirófano. En el caso del proceso de control de los instrumentos y de su introducción en las cajas, donde se han de esterilizar, se pueden dar también grados de complejidad mayor pues, aunque es básicamente lineal como proceso, puede haber simultaneidades de tareas.

Las instancias cognitivas han de ponerse en juego en ambos procesos pues ninguno de los dos es totalmente rutinario y mecánico. El conocimiento, externalizado que se manifiesta en los procesos de las Consultas Externas por sus representaciones, es diverso y complejo por superposición: al mismo tiempo, o casi, se factura, se organiza la atribución del paciente al médico o a tipos de análisis, se dan horas de visita, se atiende afectivamente o consuela. En Esterilización hay diferencias entre los procesos. Algunos de ellos necesitan más grado de proyección e intervención de las instancias cognitivas como son las representaciones externas y las tomas de decisión sobre todo en los procesos de control como los de llenar cajas, identificar a la responsable.

\subsection{Las competencias y la invisibilidad de la carga de trabajo}

El concepto de competencia se refiere al conjunto de conocimientos, capacidades y actitudes cognitivas o aplicadas movilizadas en la situación y contexto de trabajo. En relación con otras nociones como conocimiento informativo, o más genéricamente, cualificación o categoría profesional, este concepto subraya la idea de que cada contexto y situación de trabajo exige una aplicación específica de estos conocimientos, capacidades, aptitudes y actitudes en su aplicación.

El trabajo invisible hace referencia al conjunto de tareas no reconocidas, tanto por parte de la empresa como en la percepción de los agentes que intervienen en los procesos y puestos de trabajo. Las razones pueden ser varias: ausencia de protocolo explicitado sobre ellas, porque van de sí en su propio ejercicio, ausencia de centralidad en los procesos, su no visibilidad física o su naturaleza borrosa o difusa. 


\section{Las Consultas Externas}

Dentro de los procesos y tareas de las Consultas Externas, la atención de la administrativa a las consultas puntuales es la que, en mayor medida, resulta invisible tanto para la organización como para las mismas administrativas. Esta tarea no está protocolada y, sin embargo, exige un elevado esfuerzo cognitivo y otras competencias por parte de las trabajadoras.

Una parte de las consultas que realizan los usuarios a las administrativas (cara a cara o telefónicamente) obligan a éstas a informarse acudiendo a las enfermeras o secretarias médicas (para lo cual deben abandonar el espacio de trabajo que ocupan en la sala de espera) o a redirigir la consulta dando un número de teléfono al que llamar o indicando el lugar del hospital al que dirigirse. Las administrativas resuelven estas tareas por el uso de las relaciones y redes informales que poseen. Una alternativa a este tipo de comportamiento consistiría en encauzar estas peticiones al punto de información en Consultas Externas, puesto que haría más visible el proceso de trabajo correspondiente, en gran medida oculto, aunque provocaría una menor satisfacción entre los usuarios. La opacidad de esta tarea proviene del hecho de que no forma parte de ningún proceso de trabajo conectado con la asistencia médica de los especialistas al contrario de lo que sucede con la programación de pruebas o la gestión de las visitas a los especialistas. Otras tareas que permanecen ocultas o difusas en el conjunto de proceso son por ejemplo dar información adecuada a las solicitudes de los pacientes, reprogramar la fecha de unos análisis, buscar alternativas a un/a paciente que ha olvidado realizar las pruebas asignadas antes de acudir al médico. En la mayoría de casos no queda ningún tipo de registro de este tipo de trabajo.

Así mismo otras tareas invisibles y sin registro o protocolo, simultaneadas con otras, se refieren a las relaciones entre las enfermeras y las administrativas, ya que reciben y dan consultas e informaciones mutuas. En algunos casos se trata de informaciones médicas directas o relacionadas con las pruebas médicas (tipos de pruebas programadas, fecha...). En otros casos son las enfermeras las que solicitan información referente a datos administrativos de los pacientes (número de teléfono, por ejemplo). No obstante el carácter invisible de dichas tareas, en todas las observaciones realizadas, la implicación fue total por parte de enfermeras y administrativas en las variadas y urgentes peticiones de pacientes para dar la información apropiada, ya fuese por medios propios, ya fuese acudiendo o llamando a terceras personas. Aunque invisibles, no pagadas ni contabilizadas, estas tareas ponen en juego capacidades emergentes no medidas ni previstas en los protocolos. La implicación y atención a los pacientes va, pues, más allá del estricto ámbito de responsabilidad y se sustenta en buena medida en "actitudes" o "capacidades" asociadas a la construcción de género, como la empatía, el trato amable o la complacencia ante solicitudes no protocoladas.

\section{El servicio de Esterilización}

El trabajo auxiliar realizado en el servicio de Esterilización constituye una condición fundamental para el funcionamiento del conjunto del hospital y concreta- 
mente de las áreas y servicios de donde procede el material "sucio" que ha de esterilizarse. El $80 \%$ de este material procede del área quirúrgica y la sala de partos, mientras que el 20\% restante procede de los dispensarios de Consultas Externas, los centros de atención primaria asociados al hospital y otras unidades del mismo.

Si bien es usual identificar un importante componente de invisibilidad en el reconocimiento del trabajo auxiliar tanto del personal administrativo como de enfermería por parte del resto del personal del Hospital, en el caso de Esterilización se da un mayor grado de invisibilidad general de todo el servicio no sólo por el lugar casi físicamente "oculto" que ocupa en los bajos del hospital, sino que además se acentúa por los estereotipos y las representaciones que de dicho servicio predominan en otros servicios hospitalarios. La idea general que se tiene de Esterilización, sobre todo desde servicios que realizan un trabajo de carácter asistencial, es que se trata de procesos de trabajo protocolados, simples y de carácter fabril. Sin embargo, las distintas sesiones de observación directa así como las entrevistas realizadas, nos han mostrado que dichas representaciones no se ajustan tanto a la realidad del trabajo efectivamente desarrollado. El subproceso de preparación del material quirúrgico, su clasificación, introducción en cajas metálicas, empaquetado, control y registro, contiene un relativo grado de complejidad. Exige intervención de la instancia cognitiva, competencias en activar representaciones, capacidad en la toma de decisiones como en las cajas devueltas por quirófanos, de reacción ante imprevistos como las peticiones por parte de quirófanos de cajas aún no preparadas, capacidad de resolución colectiva de los problemas como las decisiones acerca del material que corresponde a algunas cajas y hasta de la organización autónoma del propio trabajo.

La importancia de las consecuencias de un fallo humano en el proceso de Esterilización obliga a que sus procesos estén altamente protocolados, en particular en la clasificación, empaquetado, control y registro de las cajas de esterilización, lo que permite dar más visibilidad a los procesos. Por ello, en Esterilización la invisibilidad se desarrolla más en los procesos de coordinación y articulación con el área de los quirófanos y también en los de prelavado y lavado del material quirúrgico. La coordinación con quirófano la realizan dos trabajadoras, normalmente de mayor antigüedad, concretamente en el caso observado coincide con las que han tenido experiencia en quirófano y conocen a las enfermeras que trabajan en el mismo. Esta comunicación es prácticamente constante y marca el ritmo y el flujo de trabajo del servicio. A partir de sus resultados es posible ir planificando el trabajo inmediato no ausente de cambios y de presiones. Además, las cajas de material, que son limitadas y se utilizan en sucesivas operaciones previa esterilización de las mismas, introducen un nuevo factor de premura y ritmo más acelerado. Con todo, esta comunicación entre ambos servicios pierde algunas veces su regularidad y al mismo tiempo puede estar jalonada de frecuentes imprevistos y urgencias que implican rápidas reordenaciones de todos los procesos y tareas. En definitiva, no obstante las exigencias de visibilidad, los procesos de Esterilización pueden tener también sus dosis de invisibilidad, acentuada por la cercanía de las tareas realizadas con los conocimientos y el saber hacer propio del trabajo doméstico y no tanto de la ciencia médica (Arborio, 2001). 


\section{Consecuencias comparativas}

Si bien la naturaleza del trabajo desarrollado en las dos áreas hospitalarias estudiadas es notablemente diferente, está presente en ambas la invisibilidad de algunos de los procesos, para las trabajadoras, las coordinadoras de los servicios y en la caso de Esterilización para otros servicios del Hospital. Como se ha señalado, la invisibilidad oculta y supone tácitamente una serie de competencias clave para el desarrollo del proceso de trabajo (Jones y Wood, 1984). En Consultas Externas se destacan las capacidades relacionadas con la empatía y trato con los pacientes; así, junto con la búsqueda de eficacia, se añaden otras consideraciones "humanitarias" y no estrictamente técnicas -englobadas en el término sentimental work (Strauss et al., 1985)-, que se consideran como competencias no cualificadas dada su consideración de talentos naturalmente femeninos (Jenson, 1989). En esta área, la atención a los usuarios es continua y las peticiones que reciben las administrativas son muy variadas. Con el fin de responder adecuadamente a las demandas planteadas por los pacientes, las administrativas movilizan información y contactos sin que dichas funciones sean de su responsabilidad. En el caso de Esterilización la capacidad relacional se circunscribe al trabajo en equipo, esto es, al trato entre la pareja de trabajadoras que se ocupan del subproceso y entre éstas y enfermera instrumentista de quirófano. Además, la resolución de los problemas tiene frecuentemente un carácter colectivo de consulta y evaluación entre las auxiliares con más experiencia del equipo.

Ambos servicios comparten el hecho de que las trabajadoras de las dos áreas observadas han de reaccionar de forma rápida y flexible ante la aparición de situaciones no previstas o "problemáticas" que se resuelven gracias a la dimensión cooperativa del trabajo desarrollado. Pero se trata de una cooperación asimétrica, pues suele ser entre trabajadores de diferente rango y desde luego no colegiado.

Como se viene afirmando, el carácter inesperado o incluso accidental de las situaciones contribuye a hacer invisibles las competencias señaladas; al ser circunstancias total o un tanto imprevisibles y de resultado contingencias o inesperadas no están "catalogadas" y asumidas como tareas propias de los procesos en ambos servicios. Star y Strauss (1999) denominan a estas tareas trabajo de articulación pues se refieren a procesos y tareas que gestionan los efectos producidos por la naturaleza distribuida del trabajo. En este tipo de tareas las competencias se atribuyen tácitamente: resolución de imprevistos, representaciones y decisiones rápidas, experiencia, etc. Esta naturalización contribuye a la invisibilidad de dichos procesos y tareas. La observación minuciosa y la grabación del proceso de trabajo las ponen de manifiesto.

La invisibilidad de dichas competencias también está relacionada con el hecho de que, en gran medida, las trabajadoras ya las tenían antes de trabajar en Consultas Externas y Esterilización, por ejemplo las adquiridas en el ámbito doméstico a través de la socialización diferencial de género existente en el trabajo domésticoreproductivo (Kergoat, 1982). Sólo ha sido necesario adaptarlas a las circunstancias y características específicas de las tareas que las requieren. 
Por otro lado, y genéricamente, se ha detectado que, en buena medida, aquellas tareas que mejor ponen de manifiesto la movilización de competencias por parte de las personas observadas son también aquellas que tienen un mayor grado de invisibilidad. Este hecho provoca una minusvaloración tanto por parte de las propias trabajadoras como por parte de personas de la dirección del tipo de competencias que es necesario aplicar para un correcto desempeño de las tareas.

\section{Reflexiones y conclusiones}

Nuestro objetivo ha consistido, primero, en examinar las características de los dos procesos de trabajo seleccionados teniendo en cuenta aspectos y elementos que tratan de poner en evidencia las situaciones interactivas, distribuidas, cognitivas y, en su caso ocultas, de los procesos de trabajo, y segundo, mostrar una metodología ad hoc para ello.

La conclusión general que se desprende del análisis es el carácter dual o incluso contradictorio que se da en los procesos de los servicios auxiliares estudiados, precisamente por estar a medio camino entre unas exigencias pretéritas pero vigentes y otras futuras, aunque incipientes, que se implantan de manera exógena y sin consulta a los/as trabajadores/as. Así, hemos visto cómo pueden darse, en un mismo $\mathrm{o}$ en diversos procesos de trabajo, situaciones y consecuencias muy diversas y controvertidas. Rememoramos algunos casos. Los/as trabajadores/as pueden tener teóricamente un grado de autonomía en la organización del desarrollo de su trabajo pero verse, al mismo tiempo, sometidos/as en su ejecución a la presión de un flujo tenso con unos ritmos completamente deterministas o que dicha autonomía se realice sólo en los intersticios de las tareas que a lo largo de la jornada y de modo contingente se establecen externamente como prioritarios. Un determinado proceso de trabajo de naturaleza serial y, por tanto, previamente programable y previsible en su linealidad, puede quedar completamente desbaratado al estar sometido a exigencias y urgencias externas. Algunos de los procesos analizados, con apariencia rutinaria producen, sin embargo, en el/la trabajador/a, un fuerte estrés -tensión y atención- que proviene de la actuación simultánea de diferentes instancias como las cognitivas, funcionales, emocionales y sociales. En algunos casos el/la trabajador/a toma decisiones de trabajo sin posibilidad de comunicarse con otros/as trabajadores/as que tienen funciones equivalentes a las suyas; sobre todo en decisiones en las que convergen responsabilidades y contenidos a veces contradictorios y exigencias simultáneas que tienen su origen en otros procesos más complejos y cualificados.

Lo que parece evidente es la dificultad de captar, y desde luego también evaluar, las competencias -y más cuanto más complejas- y la invisibilidad de las tareas cuando se hace exclusivamente desde fuera de los procesos, situaciones y contextos en que se realizan, y más con indicadores prefijados unilateral o parcialmente por una parte de los agentes implicados en los procesos de trabajo.

Muchas de las exigencias contradictorias aludidas, aunque diferentemente en cada servicio, pueden ser la causa del estrés físico y mental y del absentismo que se da 
en los dos servicios. Además gran parte de las competencias, instancias personales y colectivas puestas en juego, formas de aprendizaje por contagio y socialización, implicaciones emotivas, responsabilidades compartidas, situaciones "problemáticas" resueltas cooperativamente, pasan totalmente desapercibidas, incluso para las propias trabajadoras. Algunas otras son solamente percibidas dentro del equipo y/o por la trabajadora pero son invisibles para los responsables de las áreas estudiadas. Son competencias in situ que no se ven reflejadas, ni reconocidas, ni legitimadas en ningún sistema de evaluación o promoción. Este hecho es en parte el resultado de los cambios introducidos en la organización del trabajo, que priman la autoorganización del trabajo en un contexto de flujo tenso; y en parte es también una consecuencia de ser competencias concebidas como no cualificadas por ser consideradas talentos naturalmente femeninos. Así, la aplicación de dichas competencias se entiende como parte rutinaria y sustantiva de su trabajo.

\section{Bibliografía}

Arborio A. M. (2001). Un personel invisible. Les aides-soignantes à l'hôpital. Paris: Antropos.

Barzelay, M. (2001). The New Public Management. Improving Research and Policy Dialogue. Berkeley: University of California Press.

Castillo, J.J.; De las Alas-Pumariño, A.; Del Bono, A.; Fernández, J; Galán, A.; Santos, M. (2000). "División del trabajo, cualificación, competencias". Sociología del trabajo, 40, 3-50.

Cicourel A.V. (1999). "The Interaction of Cognitive and Cultural Models in Health Care Delivery". En Sarangi, S y Roberts, R (eds.). Talk, Work and Institutional Order: Discourse in Medical, Mediation and Management Setting (pp. 183-224). Berlin-New York: Mouton de Gruyter.

Cicourel, A.V. (2007). "La gestión de las visitas en un servicio médico especializado: la organización y la comunicación en un régimen de 'sobrecarga' cognitiva". En Lozares (ed.) Interacción, redes sociales y ciencias cognitivas (pp.17-50). Granada: Editorial Comares.

Crompton, R. (1997). Women and Work in Modern Britain. New York: Oxford University Press.

Conein B. y Jacopin E. (1994). "Action Située et cognition, le savoir en place", Sociologie du Travail, 4, 475-500. 
De Terssac, G. (1995). Autonomía en el trabajo. Madrid: Ministerio de Trabajo y Seguridad Social.

Dunleavy, P. (1994). "The Globalization of Public Services Production: Can Government Be Best in World?". Public Policy and Administration, 9 (2), 36-64

Durand, J.P. (2001). "Travail informationnel et flux tendu". En Durand, C. y Pichon, A. (eds). Temps de travail et temps libre. Brussels: De Boeck.

Durand, J.P. (2004). La chaîne invisible. Travailler aujourd'hui: flux tendu et servitude volontaire. Paris: Seuil.

Elson, D. y Pearson, R. (1981). "Nimble fingers make cheap workers: An analysis of women's employment in Third World manufacturing". Fememinist Review, 7, $87-107$.

Gallego, R. (2002). “¿Actores o instituciones? La política sanitaria catalana”. En Grau, M. y Mateos, Análisis de políticas públicas en España: enfoques y casos. Valencia: Tirant lo Blanch.

Hood, C. (1994). Explaining Economic Policy Reversals. Buckingham: Open University Press.

Hutchins E. (1995). "How a Cockpit Temembers its Speeds". Cognitive Science, 19, 265-288.

Hutchins E. y Klausen T. (1996). "Distributed cognition in an airplane cockpit" en Engeström Y., Middleton D. Cognition and communication at work (pp. 15-33). Cambridge: University Press.

Jenson, J. (1989). "The talents of women, the skills of men: flexible specialization and women". En Wood, S. (ed.). The transformation of work? Skill, flexibility and the labour process. Londres: Unwin Hyman.

Jones, B. y Wood, S. (1984). "Qualifications tacites, division du travail et nouvelles technologies”. Sociologie du Travail, 26 (4), 407-420.

Kergoat, D. (1982). Les Ouvrières. Paris: Le Sycomore.

Kergoat, D. (1994). "Por una sociología de las relaciones sociales. Del análisis crítico de las categorías dominantes a una nueva conceptualización". En Borderias, C.; Carrasco, C; Alemany, C. (eds). Las mujeres y el trabajo: Rupturas conceptuales. Barcelona: FUHEM-Icaria. 
Khöler, C.; Woodard, J. (1997). "Systems of Work and Socio-Economic Structures: A Comparison of Germany, Spain, France and Japan". European Journal of Industrial Relations, 3 (1), 59-82.

Lichtenberger, Y. (2003). "Compétence, compétences". En: Allouché, J. (coord.). Encyclopédie des ressources humaines (pp. 203-215). Paris : Éditions Vuibert.

Lozares, C. (2000). "L'activitat situada i/o el coneixement socialment distribuït en l'estudi d'un lloc de treball". Revista Catalana de Sociologia. 11, 175-182.

Lozares, C. (2001). "La actividad situada y/o el conocimiento socialmente distribuido". Papers, 62, 97-131.

Lozares, C. (2007a). "Aproximación a la cognición socialmente distribuida, CSD: un caso de estudio". En Lozares, C. (ed.). Interacción, Redes Sociales y Ciencias Cognitivas (pp. 267-312). Granada: Comares.

Lozares, C. (2007b). "La unidad de análisis, sus agentes y contextos y los modos de interacción en los procesos de trabajo: un estudio de caso". Papers, 83, 97-122.

Lozares, C; Verd, J.M.; Moreno, S.; Barranco, O.; Massó, M. (2004). "El proceso de trabajo desde las perspectivas de la Actividad Situada y del Conocimiento Socialmente Distribuido". Cuadernos de Relaciones Laborales, 22, 67-87.

Massó, M. (2011). "La individualización de la relación de empleo en las grandes empresas en España. El caso de la industria del automóvil y el sector sanitario".Revista Internacional de Sociología, 69, 1, 143-166.

Messing, K. (1998) "Hospital Trash: Cleaners speak of their role in disease prevention". Medical Anthropology Quarterly, 12 (2): 168-187.

Olías de Lima, B. (1999). "Empleo y relaciones laborales en la administración pública". En Miguélez, F. y Prieto, C. (dir. y coord.). Las relaciones laborales en España. Madrid: Siglo XXI.

Paradeise, C. y Lichtenberger, Y. (2001). “Compétence, compétences”. Sociologie du travail, 43 (1), 33-48.

Pastor, I. (2004). "Cambios en los servicios sanitarios públicos: Los efectos de la gestión privada en las condiciones de trabajo". Comunicación presentada en el VIII Congreso Español de Sociología. 
Pastor, I. (2008). "La reforma del sector sanitario en Cataluña en los últimos 20 años. El caso de los servicios sanitarios hospitalarios de la Generalitat de Cataluña", RIO: Revista Internacional de Organizaciones, 1, 55-75.

Peneff, J. (1998). "Medida y control de las observaciones en el trabajo de campo. El ejemplo de las profesiones del sector servicios". Sociología del Trabajo, 33, 3-25.

Pinilla, J. (2004). "Intensificación del esfuerzo de trabajo en España". Cuadernos de Relaciones Laborales, 22, 2, 117-135.

Sala, G. y Planas, J. (2009) "Retos teóricos e implicaciones metodológicas del enfoque de competencia laboral". Sociología del Trabajo, 66, 31-46.

Star, S. L. y Strauss, A. (1999) "Layers of Silence, Arenas of Voice: The Ecology of Visible and Invisible Work". Computer Supported Cooperative Work, 8, 9-30. Verd, J.M.; Barranco, O.; Moreno, S. (2007). "El análisis de los procesos de trabajo mediante métodos etnográficos: el caso del trabajo administrativo de consultas externas hospitalarias", Papers, 83, 145-168.

Strauss A.; Fagerhaugh, S.; Suczek, B. y Wiener, C. (1982). "Sentimental work in the technologized hospital". Sociology of health and illness Vol 4. № 3: 254- 277.

Suchman L. (1993). "Respuesta a la accion situada de Vera y Simon: una intepretacion simbólica". Cognitive Science, 17, 71-75. 